\title{
COUNTERING OVERSEGMENTATION IN PARTITIONING-BASED CONNECTIVITIES
}

\author{
Georgios K. Ouzounis, Michael H.F Wilkinson \\ Institute for Mathematics and Computing Science \\ University of Groningen \\ P.O.Box 800, 9700 AV Groningen \\ The Netherlands
}

\begin{abstract}
A new theoretical development is presented for handling the over-segmentation problem in partitioning-based connected openings. The definition we propose treats singletons generated with the earlier method, as elements of a larger connected component. Unlike the existing formalism, this new method allows detection of filamentous structures linking larger objects. This is demonstrated in attribute filtering on neuron images.
\end{abstract}

\section{INTRODUCTION}

The notion of connectivity in images characterizes the way pixels are grouped to form connected components or flatzones in gray-scale. In mathematical morphology connected filters [1] have been developed which perform filtering based on these groupings. Connected filters modify the intensity of connected image regions instead of individual pixels. This prevents edge distortion, a property highly desirable in may applications. Connected filters are usually defined by 4- or 8-way connectivity (in 2-D). However, several theoretical developments concerning generalizations of the notion of connectivity have been proposed, aiming to improve the robustness and increase the versatility of these filters. An example is second-order connectivity $[2,3]$ which is an extension to conventional connectivity providing a flexible approach in handling groups of connected sets as single clusters or object regions bridged by narrow elongated segments as individual sets. These two conditions are referred to as clustering and partitioning respectively. The connectivity generalization given by means of a connected opening and a generalizing operator $\psi$ yields two separate definitions that distinguish between the two cases. Our study focuses on the over-segmentation problem generated from partitioning operators when considering image regions that bridge the connected sets of interest. Note that this is not considered a problem if the operators aim at extracting grains of the

This work is funded by the Netherlands Organization for Scientific Research under project number 612.065.202.
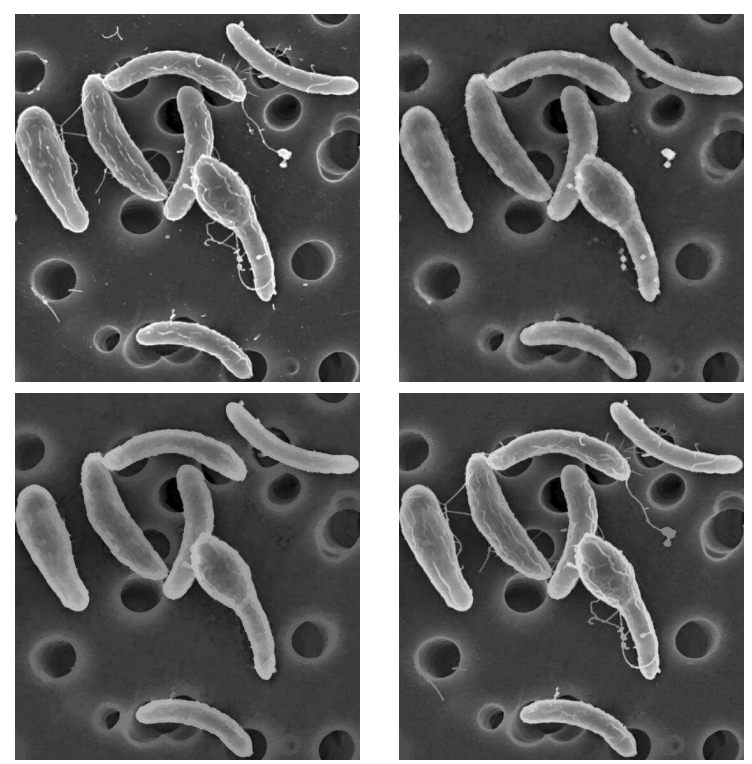

Fig. 1. Example of oversegmentation - Escherichia coli: original image; connectivity map obtained by opening with a 9x9 SE; area opening using the Dual Input Max-Tree (attribute threshold of 500); area opening using conventional Max-Tree (attribute threshold 500).

image objects [4]. An example is illustrated in Fig.1 where the Dual Input Max-Tree algorithm [5] is employed for extracting bacteria merged by thin elongated structures. The resulting image appears blurred due the removal of all singletons primarily responsible for image sharpness.

\section{THEORY}

\subsection{Connectivity and Connected Openings}

Let $E$ be a universal (non-empty) set and $\mathcal{P}(E)$ the collection of all subsets of $E$. A set $X$ representing a binary image is a subset of $\mathbf{M}$, the image domain, which in turn is a subset of $E$. We say that $X$ is connected if it cannot be partitioned 

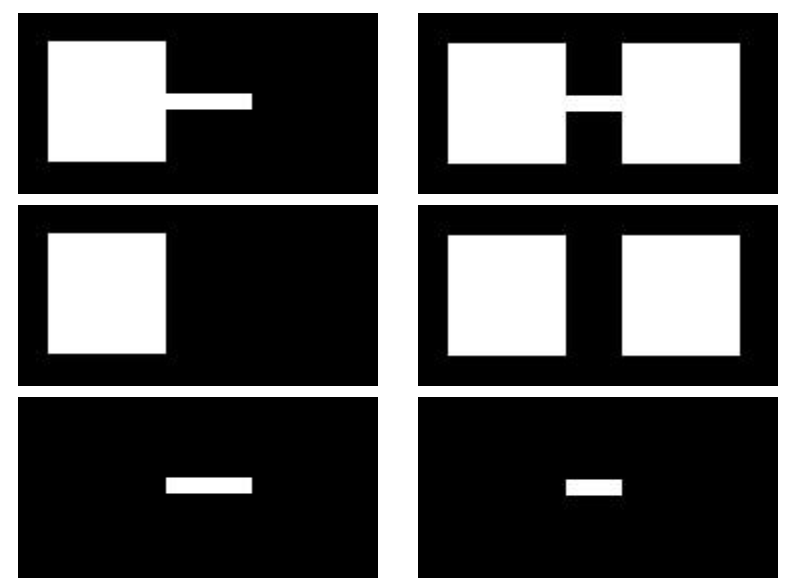

Fig. 2. Example of sets failing increasingness of $\Gamma_{x}^{\psi}$ according to def. 3: original image $X$ (top left); $Y$ : a superset of $X$ (top right); connectivity map $\psi(X)$ (middle left); connectivity map $\psi(Y)$ (middle right); set difference $X \backslash \psi(X)$ (bottom left); set difference $Y \backslash \psi(Y)$ (bottom right).

into two non-empty closed or opened sets. The connectivity in $\mathbf{M}$ is represented by a family $\mathcal{C}$, the connectivity class [6] which is defined accordingly:

Definition 1 f family $\mathcal{C} \subseteq \mathcal{P}(E)$ with $E$ an arbitrary nonempty set, is called a connectivity class if it satisfies:

1. $\emptyset \in \mathcal{C}$ and $\{x\} \in \mathcal{C}$ for $x \in E$,

2. if $C_{i} \in \mathcal{C}$ with $i=1, \ldots N$ and $\bigcap_{i=1}^{N} C_{i} \neq \emptyset$, then $\bigcup_{i=1}^{N} C_{i} \in \mathcal{C}$

This means that both the empty set and singletons are connected, and any union of connected sets which have a nonempty intersection is connected. Any image $X$ is composed of a number of connected components or grains $C_{i} \in \mathcal{C}$, with $i$ from some index set $I$. For each $C_{i}$ there is no set $C \supset C_{i}$ such that $C \subseteq X$ and $C \in \mathcal{C}$. If a set $C$ is a connected component of $X$ we denote this as $C \lessdot X$.

The notion of connectivity can be alternatively defined by means of connected openings [2,6]. Connected openings are operators acting on connected components instead of individual pixels and are defined as follows.

Definition 2 The binary connected opening $\Gamma_{x}$ of $X$ at point $x \in \mathbf{M}$ is given by

$$
\Gamma_{x}(X)=\left\{\begin{array}{lr}
C_{i}: x \in C_{i} \wedge C_{i} \lessdot X & , \text { if } x \in X \\
\emptyset & \text {, otherwise. }
\end{array}\right.
$$

Thus $\Gamma_{x}$ extracts the connected component to which $x$ belongs, discarding all others.

\subsection{Second-Order Connectivity}

In this section we only present the partitioning-based connectivity generalization. For an extensive discussion the reader is referred to $[2,7]$. Connected sets emerging from this generalization, referred to as contracted, are given by the following connected opening:

Definition 3 Let $\psi$ be an increasing, idempotent and antiextensive operator and $X$ a subset of $E$. The partitioning based connected opening associated with the second-order connectivity class $\mathcal{C}^{\psi}$ is given by:

$$
\Gamma_{x}^{\psi}(X)= \begin{cases}\Gamma_{x}(\psi(X)) & \text { if } x \in \psi(X) \\ \{x\} & \text { if } x \in X \backslash \psi(X) \\ \emptyset & \text { otherwise }\end{cases}
$$

where $\psi(X)$ is referred to as the connectivity map. This transformation yields the same image $X$ characterized by a connectivity class $\mathcal{C}^{\psi}$ that is a subset of the original

$$
\mathcal{C}^{\psi} \subseteq \mathcal{C}
$$

Elements discarded by the operator $\psi$ are interpreted by $\Gamma_{x}^{\psi}(X)$ as singletons. Therefore the image $X$ breaks up to a number of connected components $C_{i}$ that are invariant to $\psi$, i.e. $\psi\left(C_{i}\right)=C_{i}, \forall i \in I_{t}$ and a set of singletons i.e. $C_{i}=\{x\}, \forall i \in I_{s}$, where $I=I_{t}+I_{s}$ the connected component index. The union of the invariant components, termed stable [7] together with the singletons and the empty set defines the partitioning-based connectivity $\mathcal{C}^{\psi}$.

\subsection{Second-Order Attribute Filters}

Binary attribute openings [8] are a subclass of connected openings [9] incorporating an increasing criterion $T$. This criterion is applied by means of a trivial opening $\Gamma_{T}$ for accepting or rejecting connected sets extracted by the connected opening. The increasingness of $T$ implies that if a set $A$ satisfies $T$ then any set $B$ such that $B \supseteq A$ satisfies $T$ as well. A common expression of $T$ is of the form

$$
T(C)=(\operatorname{Attr}(C) \geq \lambda)
$$

with $\operatorname{Attr}(C)$ some real-valued attribute of $C$, and $\lambda$ the attribute threshold. The trivial opening $\Gamma_{T}$ is defined as follows $\Gamma_{T}: \mathcal{C} \rightarrow \mathcal{C}$ operating on $C \in \mathcal{C}$ yields $C$ if $T(C)$ is true, and $\emptyset$ otherwise. Furthermore, $\Gamma_{T}(\emptyset)=\emptyset$. An example of an increasing attribute filter is the area opening which is illustrated at the bottom right image in Fig.1 (gray-scale).

The second-order binary attribute opening involves the trivial opening $\Gamma_{T}$ on the output of a generalizing connected opening $\Gamma_{x}^{\psi}(X)$ and is defined as follows: 
Definition 4 The second-order binary attribute opening $\Gamma_{\psi}^{T}$ of a set $X$ with increasing criterion $T$ is given by:

$$
\Gamma_{\psi}^{T}(X)=\bigcup_{x \in X} \Gamma_{T}\left(\Gamma_{x}^{\psi}(X)\right)
$$

The second-order binary attribute opening is equivalent to performing a trivial opening on all connected components of the intersection between the original image and the connectivity map. Note that if $T$ is non-increasing we have an attribute thinning rather that an attribute opening. An example is the scale-invariant elongation criterion of the form (4), in which $\operatorname{Attr}(C)=I(C) / A^{2}(C)$, with $I(C)$ the moment of inertia of $C$ and $A(C)$ the area [10].

\section{COUNTERING OVER-SEGMENTATION}

It has been shown previously that partitioning-based connectivity given by the connected opening of (2) causes severe oversegmentation [11] due to the treatment of regions $X \backslash \psi(X)$ in (2b). This leads to the unfortunate situation that an attribute opening based on a partitioning connectivity [5] is equivalent to the standard attribute opening applied to $\psi(X)$ (Fig.1). To counter this we propose a different definition, which, rather than considering all pixels $x \in X \backslash \psi(X)$ as singletons, considers them members of the connected components (according to $\mathcal{C}$ ) of $X \backslash \psi(X)$. We arrive at

Definition 5 The second-order, partitioning-based connected opening $\Gamma_{x}^{\psi(X)}(X)$ of an image $X$ given a modified replica by an increasing, idempotent and anti-extensive operator $\psi$, is defined as

$$
\Gamma_{x}^{\psi(X)}(X)= \begin{cases}\Gamma_{x}(\psi(X)) & \text { if } x \in \psi(X) \\ \Gamma_{x}(X \backslash \psi(X)) & \text { if } x \in X \backslash \psi(X)(6 \mathrm{a}) \\ \emptyset & \text { otherwise }\end{cases}
$$

The first step is to show that $\Gamma_{x}^{\psi(X)}(X)$ is a connected opening defining the connectivity class $\mathcal{C}^{\psi(X)}$. To verify this, $\Gamma_{x}^{\psi(X)}(X)$ must be increasing, idempotent and anti-extensive. Note that we suggest a connectivity class associated with $\psi(X)$ as opposed to an operator $\psi$. The reason for this follows from the increasingness requirement which is explained below.

The anti-extensiveness of the operator (6) follows from the fact that in each case of $(6) \Gamma_{x}^{\psi(X)}(X) \subseteq X$. To prove increasingness and idempotence, we only need to investigate $(6 \mathrm{~b})$, because the other two cases are identical to (2a) and (2c) and the proof of $\Gamma_{x}^{\psi}(X)$ given in [2] applies to $\Gamma_{x}^{\psi(X)}(X)$ for those two cases. In the case of (6b), $x \notin$ $\psi(X)$. Let $X \subseteq Y$, and $x \in X \backslash \psi(X)$. Using an operator based connectivity

$$
\Gamma_{x}^{\psi(X)}(X)=\Gamma_{x}(X \backslash \psi(X))
$$

and

$$
\Gamma_{x}^{\psi(X)}(Y)=\Gamma_{x}(Y \backslash \psi(Y))
$$

This does not guarantee $X \backslash \psi(X) \subseteq Y \backslash \psi(Y)$ and an example is illustrated in Fig.(2). Alternatively, selecting a connectivity class given a connectivity map $\psi(X)$, equation (7) remains as is but (8) becomes

$$
\Gamma_{x}^{\psi(X)}(Y)=\Gamma_{x}(Y \backslash \psi(X))
$$

Obviously, $X \backslash \psi(X) \subseteq Y \backslash \psi(X)$, and because $\Gamma_{x}$ is increasing, $\Gamma_{x}(X \backslash \psi(X)) \subseteq \Gamma_{x}(Y \backslash \psi(X))$, which means that $\Gamma_{x}^{\psi(X)}(X) \subseteq \Gamma_{x}^{\psi(X)}(Y)$, proving increasingness.

Idempotence in the case of (6b) is proven by considering

$$
\Gamma_{x}^{\psi(X)}\left(\Gamma_{x}^{\psi(X)}(X)\right)=\Gamma_{x}^{\psi(X)}\left(\Gamma_{x}(X \backslash \psi(X))\right)
$$

for any $x \in X \backslash \psi(X)$. Because $x \in \Gamma_{x}(X \backslash \psi(X))$ in this case and

$$
\Gamma_{x}(X \backslash \psi(X)) \backslash \psi(X)=\Gamma_{x}(X \backslash \psi(X)),
$$

we have that

$$
\begin{aligned}
\Gamma_{x}^{\psi(X)}\left(\Gamma_{x}(X \backslash \psi(X))\right) & =\Gamma_{x}\left(\Gamma_{x}(X \backslash \psi(X))\right) \\
& =\Gamma_{x}(X \backslash \psi(X)),
\end{aligned}
$$

through the idempotence of $\Gamma_{x}$. Combined with (10) this proves idempotence. Therefore, $\Gamma_{x}^{\psi(X)}(X)$ is indeed an opening. We now need to prove that $\mathcal{C}^{\psi(X)}$ is a connectivity class. To this extent, for the connected sets $C_{i}$ it must be shown that

$$
\bigcap_{i} C_{i} \neq \emptyset \Rightarrow \bigcup_{i} C_{i} \in \mathcal{C}^{\psi(X)} .
$$

Let $x$ be an image element such that $x \in \bigcap_{i} C_{i}$ which must exist if $\bigcap_{i} C_{i} \neq \emptyset$. The proof in the case that $x \in \psi(X)$ is identical to that given for $\mathcal{C}^{\psi}$, so we will focus on the case that $x \notin \psi(X)$. Because each $C_{i}$ is connected according to $\mathcal{C}^{\psi(X)}$, we have that

$$
\Gamma_{x}^{\psi(X)}\left(C_{i}\right)=\Gamma_{x}\left(C_{i} \backslash \psi(X)\right)=C_{i},
$$

for all $C_{i}$. This means that each $C_{i}$ is a connected component of $E$ according to the underlying connectivity class $\mathcal{C}$, with the restriction that $C_{i} \cap \psi(X)=\emptyset$. Thus, because all $C_{i} \in \mathcal{C}$, and $\bigcap_{i} C_{i} \neq \emptyset$, we have that $\bigcap_{i} C_{i} \in \mathcal{C}$. This implies that

$$
\Gamma_{x}\left(\bigcup_{i} C_{i}\right)=\bigcup_{i} C_{i}
$$

and because all $C_{i} \cap \psi(X)=\emptyset$

$$
\Gamma_{x}\left(\bigcup_{i} C_{i} \backslash \psi(X)\right)=\bigcup_{i} C_{i}
$$

which for $x \notin \psi(X)$ means that

$$
\Gamma_{x}^{\psi(X)}\left(\bigcup_{i} C_{i}\right)=\bigcup_{i} C_{i},
$$

proving (13), and therefore $\mathcal{C}^{\psi(X)}$ is a valid connectivity class. 

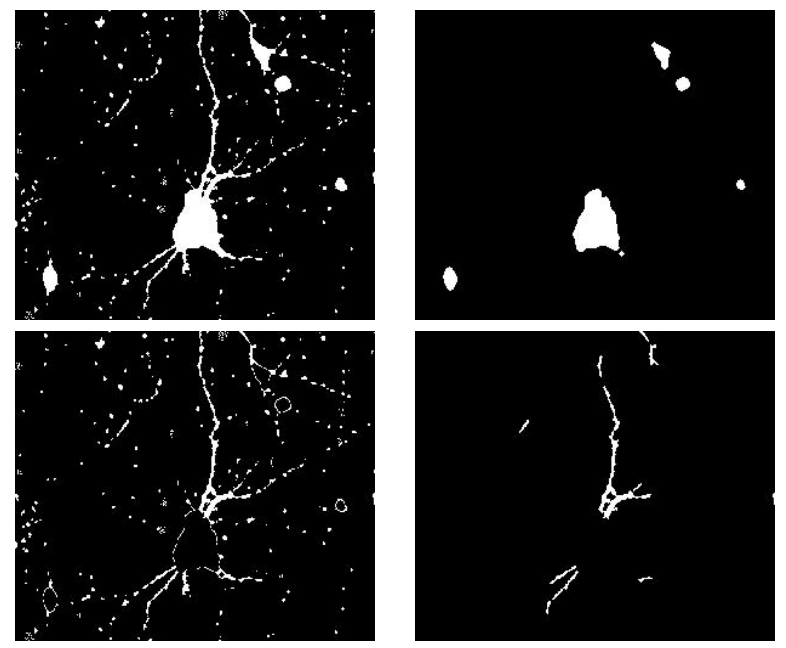

Fig. 3. Attribute thinning of neurons: original image $X$ (top left); connectivity map $\psi(X)$ given by an opening with a ball SE of radius 3 (top right); the difference image corresponding to $X \backslash \psi(X)$ (bottom left); elongation filtering on $X \backslash \psi(X)$ and also overall filter output (bottom right).

\section{APPLICATION TO ATTRIBUTE FILTERING}

This novel type of connectivity generalization based on a given connectivity map can be demonstrated with an attribute filtering example. Consider the image of neurons in Fig.3. The aim here is to isolate the dendrites from the soma (cell body) and remove the noise. We proceed by computing the elongation measure and threshold for an attribute value of 2. Combining equations (5) and (6) we derive:

$$
\Gamma_{\psi(X)}^{T}(X)=\Gamma^{T}(\psi(X)) \cup \Gamma^{T}(X \backslash \psi(X))
$$

Thus, given the connectivity map $\psi(X)$, we filter the set difference $X \backslash \psi(X)$ and the map $\psi(X)$ and then take their union. The elongation measure of the connected components in the connectivity map is below the attribute threshold therefore the resulting image is empty. The filter output consequently yields the same image as the attribute thinning of the set difference.

\section{DISCUSSION}

In section 3 we showed that over-segmentation in binary images can be dealt with extracting connected components corresponding to regions in $X \backslash \psi(X)$ instead of singletons according to definition 3 . This allows us to assign meaningful attributes to these structures and therefore filter second-order connected sets without disturbing the edges. The application we demonstrate in section 4 only shows the strength of this method compared to the previous, which so far is only applicable in binary images. Our contribution is primarily the mathematical framework since similar results can be obtained by other morphological methods. To this extent we present an operator (definition 5) producing a valid connectivity generalization associated with a connectivity map rather than an operator. We are currently working on extensions of this theory, gray-scale generalizations and algorithms for their efficient computation.

\section{REFERENCES}

[1] P. Salembier and J. Serra, "Flat zones filtering, connected operators, and filters by reconstruction," IEEE Trans. Image Proc., vol. 4, pp. 1153-1160, 1995.

[2] C. Ronse, "Openings: Main properties, and how to construct them," Tech. Rep., Universit Louis Pasteur, Strasbourg, 1990.

[3] J. Serra, "Connections for sets and functions," in Fundamenta Informaticae, 2000, vol. 41, pp. 147-186.

[4] C. Tzafestas and P. Maragos, "Shape connectivity: Multiscale analysis and application to generalized granulometries," Journal Math. Imaging and Vision, vol. 17, pp. 109-129, 2002.

[5] G. K. Ouzounis and M. H. F. Wilkinson, "Secondorder connected attribute filters using max-trees," Proc. Int. Symp. Math. Morphology (ISMM) 2005, pp. 65-74, 2005.

[6] J. Serra, Image Analysis and Mathematical Morphology. II: Theoretical Advances, Academic Press, London, 1988.

[7] U. Braga-Neto and J. Goutsias, "Connectivity on complete lattices: New results," Comp. Vis. Image Understand., vol. 85, pp. 22-53, 2002.

[8] E. J. Breen and R. Jones, "Attribute openings, thinnings and granulometries," Comp. Vis. Image Understand., vol. 64, no. 3, pp. 377-389, 1996.

[9] H. J. A. M. Heijmans, "Composing morphological filters," IEEE Trans. Image Proc., vol. 6(5), pp. 713723, 1997.

[10] E. R. Urbach and M. H. F. Wilkinson, "Shape-only granulometries and grey-scale shape filters," in Proc. Int. Symp. Math. Morphology (ISMM) 2002, 2002, pp. 305-314.

[11] M. H. F. Wilkinson, "Attribute-space connected filters," Proc. Int. Symp. Math. Morphology (ISMM) 2005, pp. 85-94, 2005. 